\title{
La circulation au sein de l'espace résidentiel de la famille. Le cas des familles de migrants colombiens en Espagne
}

Circulation within the Family Residential Area. The Case of Colombian Migrants' Families in Spain

Circulación en el espacio residencial de las familias. El caso de las familias de inmigrantes colombianos en España

\section{Celio Sierra-Paycha}

\section{OpenEdition}

\section{Journals}

Édition électronique

URL : https://journals.openedition.org/remi/7591

DOI : 10.4000/remi.7591

ISSN : $1777-5418$

Traduction(s) :

Circulation within the Family Residential Area: The Families of Colombian Migrants in Spain - URL :

https://journals.openedition.org/remi/8784 [en]

Éditeur

Université de Poitiers

Édition imprimée

Date de publication : 1 mars 2016

Pagination : 183-209

ISBN : 979-10-90426-27-6

ISSN : 0765-0752

Référence électronique

Celio Sierra-Paycha, « La circulation au sein de l'espace résidentiel de la famille. Le cas des familles de migrants colombiens en Espagne », Revue européenne des migrations internationales [En ligne], vol. 32 $n^{\circ} 1$ | 2016, mis en ligne le 01 mars 2018, consulté le 15 avril 2022. URL : http:// journals.openedition.org/remi/7591 ; DOI : https://doi.org/10.4000/remi.7591 


\section{La circulation au sein de l'espace résidentiel de la famille. Le cas des familles de migrants colombiens en Espagne}

\section{Celio Sierra-Paycha ${ }^{1}$}

L'interaction entre les deux objets de recherche que sont la "famille " et la " migration " est fructueuse à plusieurs titres.

D'une part, la famille informe sur la migration individuelle. II est en effet pertinent de caractériser la migration d'un individu non pas comme un simple transfert de son lieu de résidence personnelle, mais de considérer comment évolue l'ensemble de son espace de vie. Classiquement, le moyen retenu pour mesurer ce phénomène est le recensement, privilégiant une assignation du migrant à un seul lieu, son lieu de résidence. Selon la définition censitaire, les migrants sont des personnes ayant effectué un transfert de "la résidence des intéressés d'un certain lieu d'origine, ou lieu de départ, à un certain lieu de destination, ou lieu d'arrivée " (Henry, 1981 : 105). Les apports des géographes et démographes (Courgeau, 1975 ; Domenach et Picouet, 1995 ; Robette, 2012) ont permis de considérer la migration, non plus comme un simple transfert de lieu de résidence, mais comme un changement au sein de l'espace de vie, c'est-à-dire comme une modification au cours du temps d'une ou plusieurs des localisations qui composent cet espace ${ }^{2}$. Or l'espace résidentiel de la famille est une composante fondamentale de l'espace de vie, bien que ces espaces ne se superposent pas, ils se recoupent bien souvent. Ainsi, Dureau et Giroud (2014) ont démontré que les lieux de résidence de la famille pouvaient structurer l'espace de vie au sein duquel les individus circulent.

D'autre part, la migration peut être conçue comme une stratégie de reproduction de la famille. Depuis Aristote, la famille a été envisagée comme unité de

\footnotetext{
1 Doctorant, Université de Poitiers, MIGRINTER (UMR 7301), Institut National d'Études Démographiques, MSHS, bât. A5, 5 rue Théodore Lefebvre,TSA 21103, 86073 Poitiers cedex 9 ; celio.sierra@gmail.com

2 Beltramone évoque à titre d'exemple quatre possibilités de changement d'espace de vie ; diffusion ou extension de l'espace de vie lorsqu'apparaît un nouveau lieu sans modification des autres localisations ; contraction lorsque disparaissent une partie des implantations ; glissement lorsqu'il y a conservation d'une partie des localisations, disparition $\mathrm{d}^{\prime}$ autres localisations et apparition de nouvelles localisations ; transplantation, lorsque disparait l'ensemble des lieux d'attache au profit de nouveaux lieux (Beltramone, 1975).
} 
reproduction économique et sociale (in Politique I, 1253b). Selon Engels (1976), qui reprend les théories aristotéliciennes, deux fonctions coexistent au sein de la cellule familiale, le travail d'une part, permettant " la production des moyens d'existence, d'objets servant à la nourriture, à l'habillement, au logement et des outils qu'ils nécessitent ", "la production des hommes eux-mêmes " d'autre part. C'est dans cette tradition marxienne que certains travaux ont envisagé la migration comme une stratégie de reproduction. Dans le cas de la migration internationale, Burawoy souligne la dissociation entre le travailleur reproductif, c'est-à-dire la famille, et le travailleur productif, c'est-à-dire le migrant (Burawoy, 1976) ; dans des contextes latino-américains, des auteurs envisagent également la migration rurale-urbaine comme une stratégie de reproduction familiale (Balán et Dandler, 1987 ; Dureau et Dupont, 1994 ; Quesnel et Del Rey, 2004). La migration a ainsi pu être envisagée comme une stratégie de diversification des activités économiques. Cette diversification permettrait en retour de faire face aux aléas économiques dans les lieux d'origine. La multi-implantation de la famille correspondrait à une évolution des logiques de reproduction familiale, marquant un passage de la " communauté territoriale " à "l'économie d'archipel "(Quesnel, 2009).

L'image de l'archipel renvoie à une famille, dont les membres seraient dispersés en îlots organisés autour d'une fonction commune de reproduction de la famille. Les études sur les dispersions familiales se sont par conséquent progressivement construites sans s'appuyer sur une vision statistique de la famille assignée au ménage et se sont donc efforcées de montrer le caractère pertinent de la prise en compte de la famille à cheval sur plusieurs lieux de résidence. La notion de système résidentiel apparaît ici comme un concept fécond pour penser l'articulation d'un ensemble de lieux de résidence familiaux. S'inspirant des contextes africains (Le Bris et al., 1987), ce concept a aussi été mobilisé dans l'analyse de contextes sud-américains (Dureau, 2004) et européens (Pfirsch, 2009 ; Dureau et Giroud, 2014). Le système résidentiel se définit par un ensemble de lieux de résidence en interaction. Tant que l'accès entre les différents pôles de l'espace résidentiel est maintenu, la dispersion de la famille n'est pas forcément un obstacle à la reproduction et constitue au contraire une ressource. Les lieux de résidence de la famille font donc système par les interactions qui se déploient entre ces lieux. Osmont (1987), à l'origine de ce concept avec Le Bris, relève la circulation des enfants, les cérémonies familiales, la transmission du patronyme, l'endogamie familiale, la solidarité matérielle et les échanges économiques entre les membres, comme ciment du système résidentiel dakarois.

Cependant, si dispersion ne signifie pas forcément segmentation, on ne saurait affirmer que dispersion implique nécessairement mutualisation des ressources à des fins de reproduction. Comme le rappelle Ma Mung (1999), la dispersion n'est pas toujours synonyme d'interaction prolongée à distance. Dans un contexte de migration internationale, la question de la segmentation des familles mérite d'autant plus d'être posée que la distance, physique et juridique ${ }^{3}$, est grande entre les lieux de résidence des familles des migrants. Les formes d'interaction à distance mises en évidence dans l'étude du transnationa-

3 En raison des politiques migratoires restrictives. 
lisme familial sont ainsi construites autour de trois grands champs de recherche, celui des remises d'argent transférées d'un lieu de résidence familial à l'autre, celui de la circulation des membres de la famille entre les lieux de résidence et enfin celui de la communication régulière à distance entre ces mêmes membres (Guarnizo, 2006).

En s'appuyant sur le cas des Colombiens en Espagne, cet article propose dans un premier temps, de faire émerger les spécificités de l'organisation de l'espace résidentiel familial des migrants, selon leurs caractéristiques sociodémographiques (sexe, catégorie sociale, année d'arrivée). Si le terme de " système résidentiel familial " n'est pas privilégié dans notre première partie, c'est que selon nous, son emploi ne se justifie que lorsque les différents lieux de résidence de la famille sont effectivement en interaction, soit par la circulation de ses membres, soit par la communication à distance entre ses membres, soit par l'envoi régulier de remises financières. Dans le cas de la migration internationale, une simple dispersion ne saurait entrainer automatiquement un fonctionnement transnational. Ce sont précisément ces trois formes d'interactions, au sein de la famille à distance, que nous analyserons dans une seconde partie où sera interrogée la validité de I'hypothèse de la non-segmentation malgré la dispersion.

Les familles de migrants internationaux colombiens installés en Espagne se prêtent bien à une telle recherche. D'une part, la Colombie connaît un grand nombre de destinations migratoires, au sein desquelles l'Espagne figure parmi les trois plus importantes, aux côtés du Venezuela et des États-Unis. D'autre part, I'Institut National de Statistiques Espagnol (INE) a réalisé une enquête quantitative sur les réseaux migratoires en Espagne en 2007, qui a notamment permis de collecter les lieux de résidence des conjoints, enfants, parents, frères et sœurs de 1029 Colombiens, nés en Colombie et représentatifs des 300000 Colombiens résidant alors sur le territoire espagnol. Bien que les anthropologues de la famille colombienne reconnaissent la grande diversité des systèmes familiaux observables en Colombie, diversité héritée en partie des différents foyers de peuplement colombien (européen, indigène et africain), ils s'accordent aujourd'hui à reconnaître la primauté de la famille nucléaire avec un rôle très important de la famille élargie dans l'organisation de la vie quotidienne (Echeverri de Ferrufino, 2004). Dans le contexte colombien, une approche de la famille définie comme l'ensemble formé par le conjoint, les enfants, les parents, mais aussi la fratrie, paraît donc pertinente.

\section{Description de la population colombienne enquêtée}

L'Encuesta Nacional de Inmigrantes (ENI) a été réalisée en 2007 dans un contexte d'accroissement sans précédent de la population migrante en Espagne et d'absence de données permettant d'explorer ce contingent de migrants arrivés essentiellement depuis le début des années 2000, en pleine croissance du marché immobilier espagnol (Pumares Fernández, García Coll et Asensio Hita, 2006). Les études sur les migrations en Espagne n'ont pas manqué de souligner la " nouveauté, la magnitude et la rapidité " du changement (Reher et Requena, 2009). En quelques années, l'Espagne est passée de pays d'émigration à terre d'immigration. Malgré tous les mouvements migratoires qui ont affecté 
I’Espagne (Colectivo IOÉ, 2012 ; Sierra-Paycha, 2014) depuis la date de cette enquête (retours, rémigrations, nouvelles immigrations), I'ENI reste une enquête sans équivalent en Europe, grâce à la taille de son échantillon (15 465 personnes dont 1029 nées en Colombie) et à son questionnaire détaillé. Ceux-ci permettent donc d'explorer en profondeur le processus migratoire en Espagne à la veille de la grande récession de 2008-2010.

La particularité des migrants colombiens en Espagne et en Europe est la féminisation du flux migratoire. Comme le montre la pyramide des âges ${ }^{4}$ (Figure 1), I'individu modal de la population colombienne installée en Espagne en 2007 est une femme ayant entre trente et trente-cinq ans. Par ailleurs, il s'agit d'une population relativement jeune et en âge de travailler.

Figure 1 : Pyramide des âges des migrants colombiens en Espagne

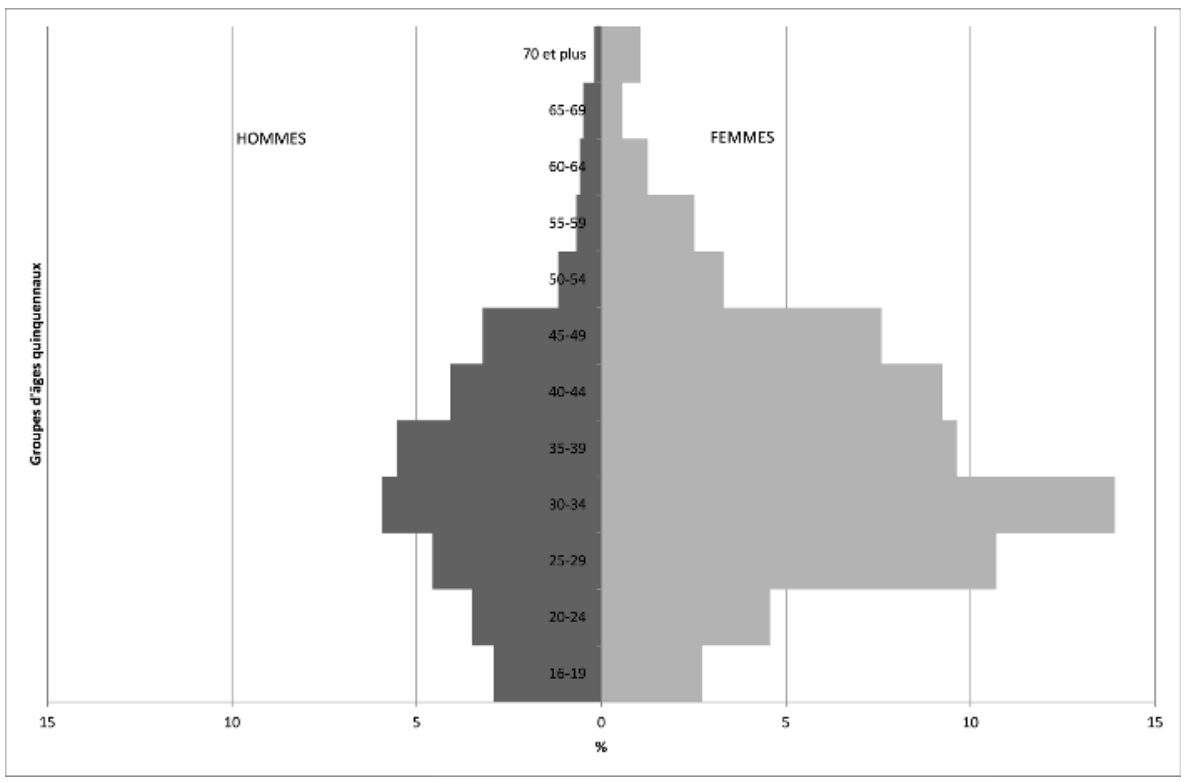

Source : ENI, $2007^{5}$.

Les Colombiens actifs vivant en Espagne en 2007 constituent une population socialement hétérogène. Des cadres et techniciens hautement qualifiés côtoient des employés non qualifiés (la moitié des femmes) et des chefs d'entreprise. On constate cependant la présence d'une classe moyenne si l'on considère le critère d'un diplôme du deuxième cycle secondaire (équivalent Baccalauréat).

4 Cette pyramide diffère de celle de Gonzalez et Echeverri pourtant construite avec la même base de données, en raison de l'application de la pondération dans leur cas et de la sélection d'une population née en Colombie, alors que nous avons choisi de travailler sur une population ayant déclaré un département de naissance en Colombie (Gonzalez et Echeverri, 2009).

5 Sauf mention contraire, tous les tableaux, graphiques et cartes de l'article proviennent de I'enquête ENI, 2007. 
La plupart des Colombiens interrogés dans I'ENI sont arrivés en Espagne depuis 1999 (plus de 80 \%). Depuis 2002 et la fin de la politique migratoire favorable de l'Espagne - pas d'obligation de visa pour les ressortissants colombiens -, les arrivées se tarissent. Les migrants arrivés récemment sont sousreprésentés dans l'enquête et on peut moins les sélectionner dans les bases de sondage de I'INE.

S'atteler à décrire la géographie résidentielle familiale implique de définir ce que nous entendons par famille. L'ENI ne collecte pas tous les lieux de résidence des liens familiaux des individus enquêtés. Si l'on connaît les lieux de résidence des enfants, des parents et des frères et sœurs vivants, le lieu de résidence du conjoint n'est pas assez détaillé. En effet, on sait si des corésidents sont des conjoints (" cónyuge o pareja " en espagnol) sans officialisation de la relation par un mariage, mais on ne sait pas si l'individu a un conjoint en dehors de son logement. En revanche, on connaît le lieu de résidence de l'époux ou de l'épouse de l'enquêté, quelle que soit la situation de cohabitation entre eux. C'est pourquoi, par abus de langage, dans cet article, par conjoint, nous entendons en réalité un époux ou une épouse avec qui l'enquêté est marié. Dans la population enquêtée (Figure 2), la proportion de personnes sans époux(se) est souvent majoritaire, quel que soit l'âge. Seuls les individus entre trente et trente-neuf ans et entre cinquante-cinq et soixante-quatre ans connaissent des pourcentages de couples mariés supérieurs à la moitié. En réalité, les proportions de concubinage sans mariage sont plus importantes. À noter que les concepteurs du questionnaire ont envisagé la forme mariée, comme l'unique forme de conjugalité pouvant être légitime sans cohabitation.

Figure 2 : Répartition des migrants colombiens selon la situation matrimoniale pour chaque groupe d'âge

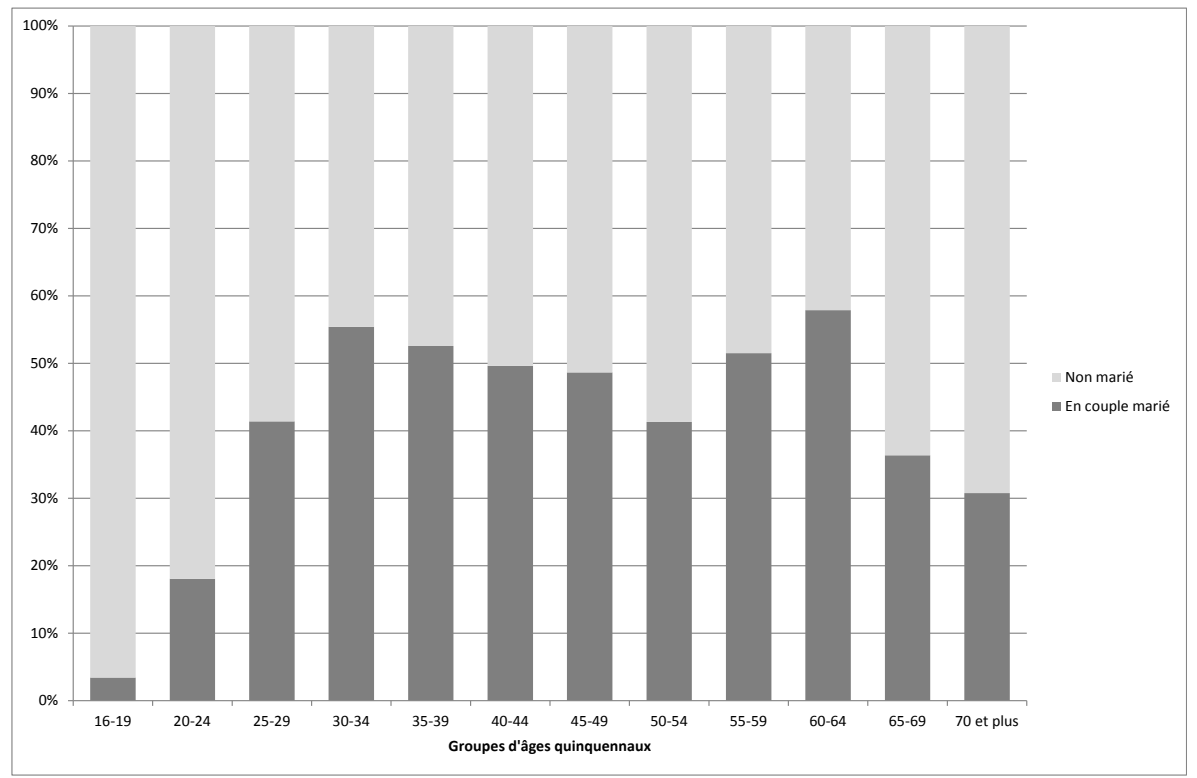


D'après le recensement colombien, la descendance finale des Colombiennes résidant en Colombie en 2005 s'élevait à 2,9 enfants par femme ${ }^{6}$ (Gonzalez et Echeverri, 2009). En Espagne, en 2007, la descendance finale des Colombiennes est légèrement inférieure, 2,27 enfants par femme, avec des variations selon le niveau d'études des femmes : 2,88 enfants par femme pour les moins qualifiées (niveau primaire ou sans études), 1,91 pour les Colombiennes ayant un diplôme d'études secondaire et 1,8 pour les Colombiennes ayant un diplôme de l'enseignement supérieur. Les mères des migrants colombiens connaissent au contraire des descendances finales plus élevées d'où la faible proportion de fils ou filles uniques dans l'échantillon (quatre-vingt-onze sur 1029 ) et la présence de familles très nombreuses (un maximum de vingt-et-un membres).

En définissant la composition de la famille par le fait d'avoir zéro, un ou deux parents vivants, des frères et sœurs, un époux ou une épouse et des enfants, vingt-quatre formes familiales sont possibles. Dans le cas des migrants colombiens installés en Espagne, le plus souvent, il s'agit de cellules familiales " d'origine ", c'est-à-dire avec au moins un parent vivant dans 888 cas sur 1029 et avec des frères et sœurs vivants dans 938 cas. Concernant les familles formées par les individus eux-mêmes, elles sont moins nombreuses, seulement 455 Colombien(ne)s ont un époux(se), mais une majorité (640) ont au moins un enfant. Par conséquent, la famille modale est une famille "d'origine " : 164 migrants enquêtés ont leurs deux parents vivants, des frères et sœurs, mais ni conjoint ni enfants. Le second type de famille le plus fréquent (143 personnes) est une famille " complète " comprenant deux parents, au moins un frère ou une sœur, un époux ou une épouse et au moins un enfant. Lorsque les personnes ont des enfants, dans la majorité des cas, ils ont aussi un époux(se), mais on relève cependant de nombreuses personnes ayant des enfants sans conjoint.

La taille du réseau familial, entendu comme la somme de l'époux(se), des enfants, des frères et sœurs et des parents vivants est variable (Figure 3) ; elle varie d'un lien familial à plus de vingt-et-une personnes. La distribution des enquêtés selon la taille de leur famille indique deux maximums locaux, à cinq personnes et à huit personnes.

En France, la taille de la parentèle diminue avec l'âge (Maison et Ortalda, 1998). Dans notre étude (Figure 4), nous observons que la taille de la famille vivante augmente au contraire globalement de seize jusqu'à cinquante-neuf ans, avant de diminuer un peu aux alentours de soixante ans. Derrière cette évolution de la taille de la famille au cours du cycle de vie, se cache également une évolution de la composition des liens familiaux. Dominé par le couple parental et la fratrie eu début de la vie active (Figure 5), la part des enfants se substitue progressivement à celle des parents. La plus grande importance de la part des enfants pour les populations les plus âgées s'explique par un effet de génération lié à une baisse globale des indicateurs de fécondité en Colombie à la fin du XXe siècle (Dureau et Florez, 1996) impliquant donc des descendances finales plus réduites pour les migrants les plus jeunes.

6 La descendance finale est le nombre moyen d'enfants mis au monde par une génération de femmes donnée tout au long de leur vie féconde, en ne tenant pas compte de leur mortalité. 
Figure 3 : Distribution des migrants colombiens en Espagne selon la taille de leur famille

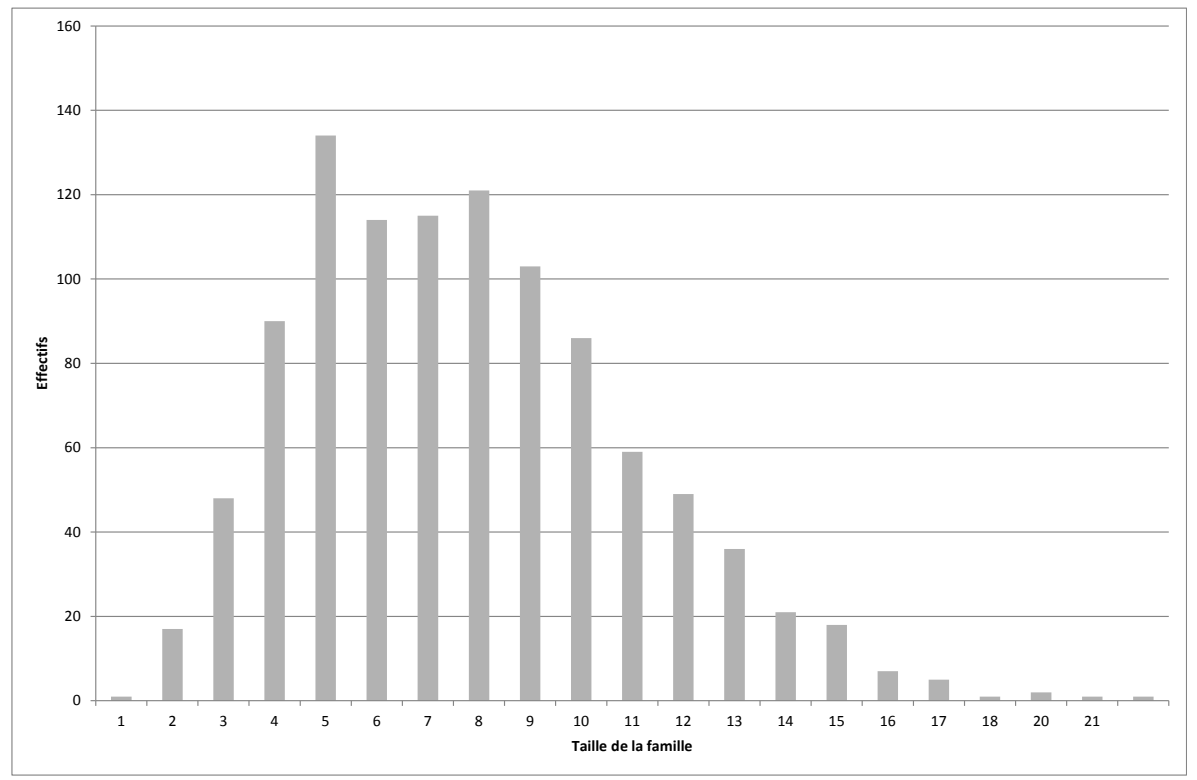

Figure 4 : Tailles moyenne et médiane du réseau familial selon l'âge

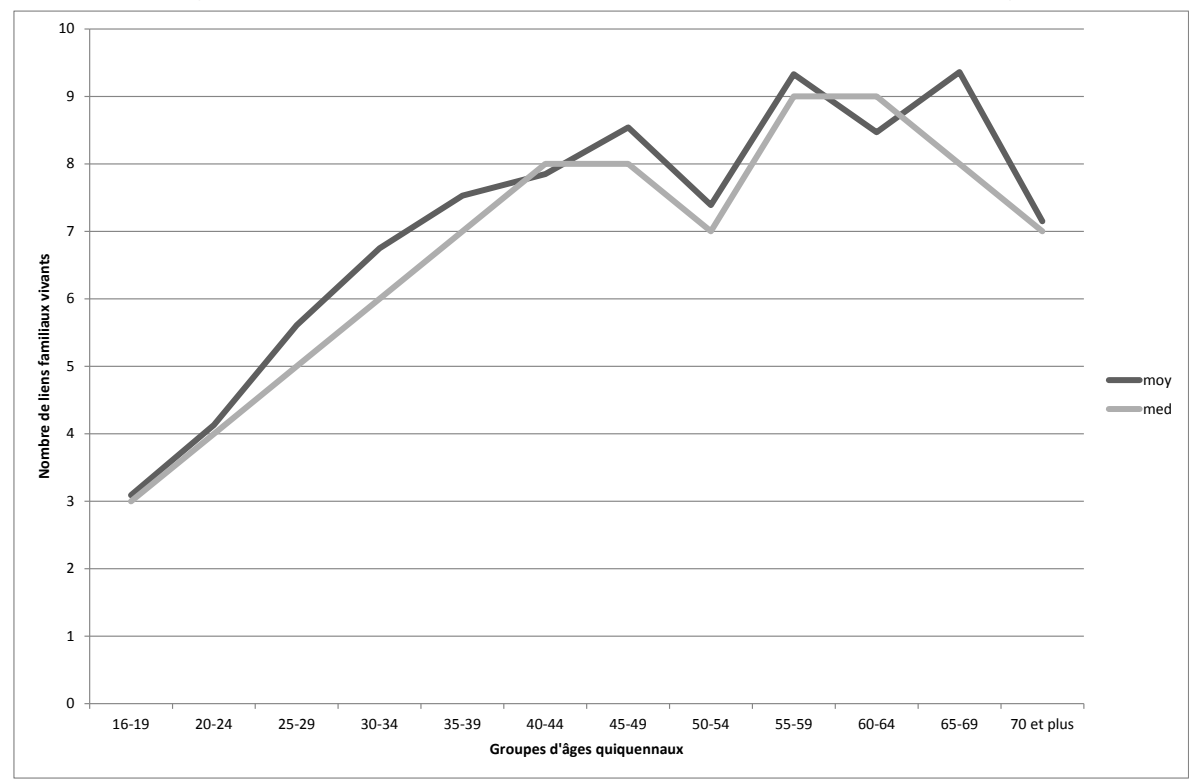


Figure 5 : Composition moyenne du réseau familial selon l'âge

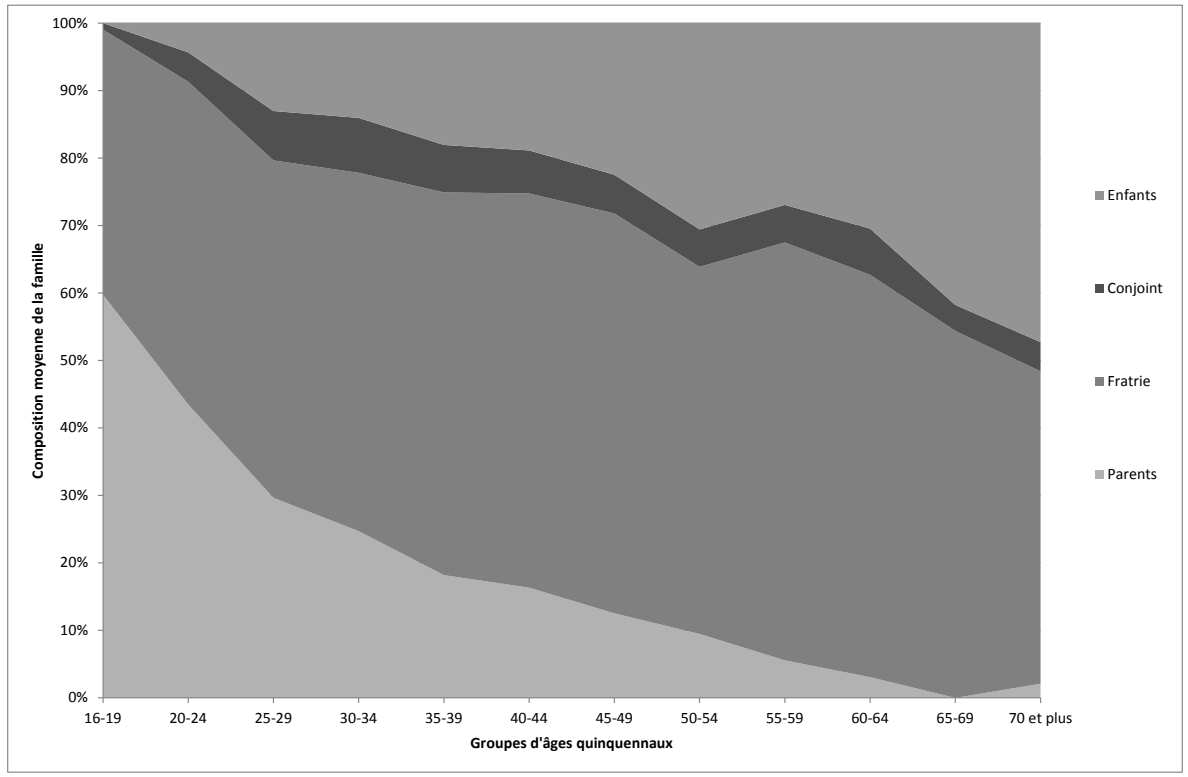

\section{Des espaces résidentiels à géométrie variable}

Cette seconde partie est consacrée à l'étude des lieux de résidence de la famille des migrants colombiens installés en Espagne en 2007. On y étudie la proximité et l'éloignement résidentiel de la famille des enquêtés. On suit ici les précautions de lecture de l'espace résidentiel familial proposées par Bonvalet et Lelièvre (2005 : 104) : "La proximité tout comme l'éloignement peuvent être le résultat d'un choix avec un mode d'être ensemble ou au contraire un désir de prise de distance vis-à-vis de la famille ". Ni forcément un choix, ni absolument une contrainte, la façon dont est organisée spatialement la famille doit plutôt "s'interpréter comme le "meilleur" arrangement possible pour accommoder le fonctionnement spécifique de chaque famille ".

\section{Une localisation résidentielle de la famille variable selon la nature des liens familiaux et l'âge du migrant}

Lorsque l'on s'intéresse globalement aux lieux de résidence de la famille des Colombiens installés en Espagne (Tableau 1), un premier constat émerge : seulement $24 \%$ de la famille est corésidente, $30 \%$ vit dans le même municipe espagnol7, $37 \%$ si I'on élargit l'échelle à I'Espagne. $63 \%$ de la famille vit donc dans un autre pays que le pays d'émigration ( $59 \%$ dans le pays d'origine et $4 \%$ dans un pays tiers). Selon la nature du lien avec l'enquêté, le taux de corésidents et le taux d'habitants en Espagne varient : $86 \%$ des conjoints, $48 \%$ des enfants, $37 \%$ des pères, $30 \%$ des mères et seulement $4 \%$ de la fratrie sont

7 Le municipe est l'entité administrative en dessous de la province, elle équivaut à la commune en France. 
corésidents. Les liens résidentiels avec le pays d'origine, que nous appellerons ici liens d'ancrage, sont surtout maintenus grâce à la résidence des parents et de la fratrie, alors que la mise en relation avec d'autres pays d'émigration colombienne semble être principalement assurée par la fratrie. Des configurations transnationales peuvent donc affecter tous les types de liens, mais avec des intensités variables selon sa nature du lien.

Tableau 1 : Répartition des lieux de résidence des membres de la famille des migrants colombiens en Espagne selon la nature du lien familial (en \%)

\begin{tabular}{|c|c|c|c|c|c|c|}
\hline $\begin{array}{l}\text { Lieux de résidence des } \\
\text { membres de } \\
\text { la famille } \\
N=1029 \text { enquêtés }\end{array}$ & Conjoint & Enfants & Père & Mère & Fratrie & Total \\
\hline \multicolumn{7}{|l|}{$\%$ en colonne } \\
\hline Espagne & 89,4 & 61,8 & 42,3 & 40,9 & 20,9 & 37,4 \\
\hline Colombie & 10,6 & 35 & 55,8 & 59,1 & 73,3 & 58,8 \\
\hline Pays tiers & - & 3,2 & 1,9 & - & 5,8 & 3,8 \\
\hline Total colonne & 100 & 100 & 100 & 100 & 100 & 100 \\
\hline \multicolumn{7}{|l|}{$\%$ en ligne } \\
\hline Corésidents & 21,9 & 34,2 & 18,5 & 16,8 & 8,6 & 100 \\
\hline Même municipe & 1,5 & 18,7 & 4,3 & 11,6 & 63,9 & 100 \\
\hline Espagne & 1,9 & 17,9 & 4,4 & 9,3 & 66,5 & 100 \\
\hline Colombie & 1,1 & 10,3 & 11,2 & 13,3 & 64,1 & 100 \\
\hline Pays tiers & - & 14,6 & 6 & - & 79,4 & 100 \\
\hline Liens familiaux & 6,1 & 17,2 & 11,9 & 13,3 & 51,5 & 100 \\
\hline Effectifs liens familiaux & 455 & 1274 & 879 & 983 & 3819 & 7410 \\
\hline
\end{tabular}

Lecture : En 2007, parmi les frères et sœurs vivants des migrants colombiens en Espagne, $21 \%$ vivent en Espagne, $73 \%$ en Colombie et $6 \%$ dans un pays tiers.

Bien sûr, la proximité résidentielle dépend de nombreux facteurs, notamment de I'âge. En France, la proximité de la parentèle augmente avec l'âge (Maison et Ortalda, 1998). La composition des corésidents varie également au cours du cycle de vie, la fratrie, surtout les parents constituent l'essentiel des corésidents de seize à vingt ans, avant que la présence des enfants et des conjoints dans le logement ne se substitue à celles des membres de la famille d'origine. À la fin de la vie active, dans le contexte francilien, ce sont les enfants qui, en décohabitant, laissent I'enquêté vivre seul avec le conjoint corésident (Golaz et Lelièvre, 2012). Cependant, dans la population colombienne étudiée (Figure 6), on observe que la part des parents parmi les corésidents ne diminue pratiquement plus à partir de vingt-cinq ans pour demeurer au-delà de $20 \%$, tandis que celle des enfants est toujours relativement importante jusqu'à soixante ans, âge auquel elle est toujours à hauteur de $10 \%$ des corésidents. 
Figure 6 : Composition moyenne du réseau familial corésident des migrants colombiens en Espagne

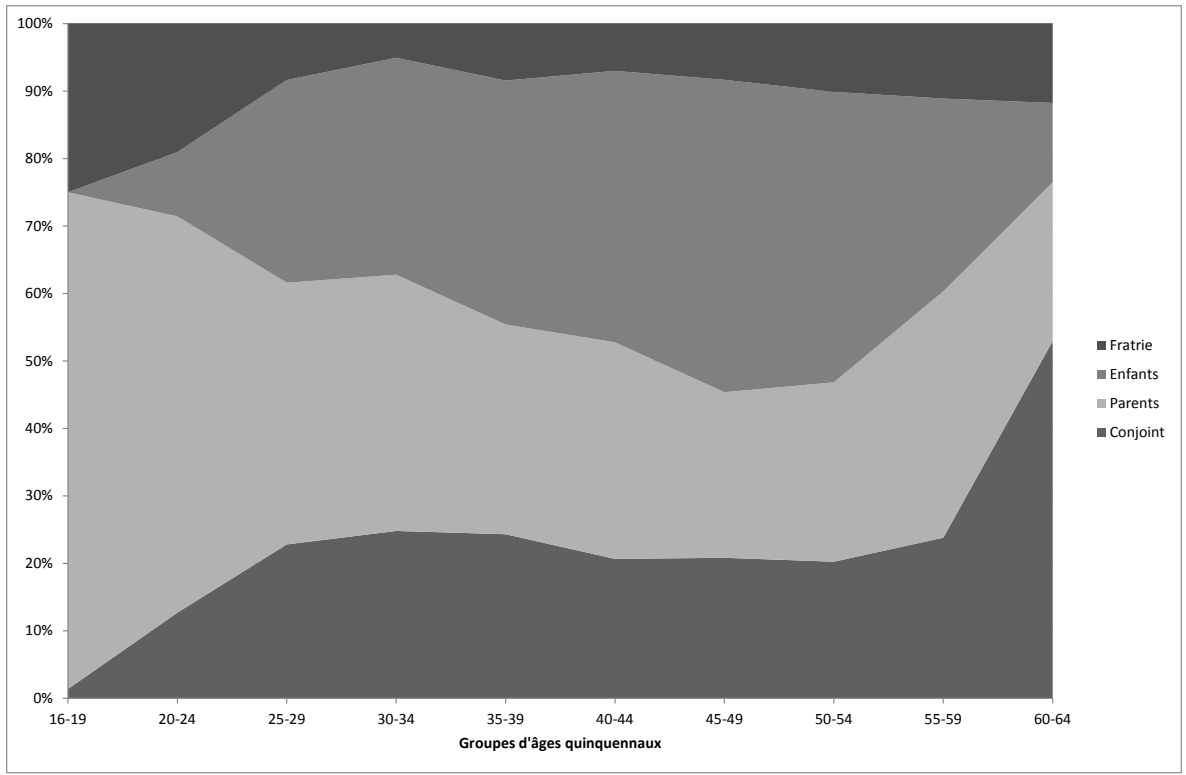

C'est autour de vingt ans, soit au début de la vie active, que la proportion de corésidents est la plus faible quel que soit le lien familial retenu. Si la proportion d'enfants corésidents et de conjoint corésident est ensuite très élevée, celle des enfants corésidents diminue au cours de la trentaine, mais à la fin de la vie active de leurs parents, ils sont encore $20 \%$ à vivre dans leur logement. Paradoxalement, la proportion des parents corésidents augmente au cours de la vie active pour atteindre un maximum local au-delà de cinquante ans.

Il apparaît ici que les espaces résidentiels familiaux sont à la fois très souvent dispersés au moins sur deux pays, mais que ceux-ci se caractérisent également par une tendance à une corésidence plutôt élevée.

\section{Des espaces résidentiels familiaux décrivant la diversité des destinations migratoires colombiennes}

Pour mieux saisir la géographie familiale avec un indicateur synthétique de la forme et non en comparant le lieu de résidence de chaque membre individuellement au lieu de résidence de l'enquêté, nous avons construit un indicateur simple montrant les pays de la dispersion résidentielle de la famille. Selon cet indicateur de la structure du réseau, les réseaux familiaux sont soit inexistants lorsque l'enquêté n'a pas de lien vivant au moment de l'enquête, soit ils sont monopolaires lorsque tous les membres vivent dans le même pays (même si ce pays est différent du pays de résidence de l'enquêté), soit ils sont bipolaires (une partie de la famille est en Espagne, I'autre en Colombie), soit ils sont multipolaires, c'est-à-dire déployés sur un espace comprenant la Colombie, l'Espagne et un pays tiers. 
Les réseaux familiaux des Colombiens installés en Espagne en 2007 sont en majorité des réseaux bipolaires (effectifs $=637 ; 62 \%$ ), les réseaux multipolaires forment le second groupe le plus nombreux $(178 ; 17 \%)$, suivi des réseaux monopolaires colombiens $(128 ; 12 \%)$ et des réseaux monopolaires espagnols $(83 ; 8 \%)$. Enfin, trois enquêtés ne connaissent le lieu de résidence d'aucun de leurs liens familiaux vivants.

178 familles sont dispersées au-delà du couple Colombie/Espagne pour des enquêtes auprès de 1026 logements dans lesquels les personnes de référence ont déclaré avoir une présence familiale en dehors du logement. $17 \%$ des familles de migrants colombiens en Espagne sont donc connectées à d'autres espaces migratoires. Ces réseaux multipolaires comprennent plusieurs milliers de liens dispersés dans vingt-cinq pays. Une distribution des pays de résidence selon le nombre de liens familiaux met en avant l'importance du " couple migratoire " dans la structuration résidentielle de ces réseaux multipolaires (la Colombie et l'Espagne apparaissent respectivement 709 fois et 510 fois comme lieux de résidence d'une partie du réseau familial des Colombiens installés en Espagne). Cette importance du couple migratoire surdétermine la distribution des lieux de résidence, mais une carte des pays de résidence hors couple migratoire (Carte 1) permet de percevoir le déploiement international des familles multipolaires. En dehors du couple migratoire, les principaux pays de résidence des liens familiaux sont des pays importants de l'histoire migratoire colombienne (123 liens résidant aux États-Unis, cinquante au Venezuela). Ailleurs, c'est essentiellement dans des pays centre-américains et européens que vit la famille des migrants colombiens en Espagne.

Carte 1 : Pays de résidence de la famille des Colombiens installés en Espagne (hors couple migratoire)

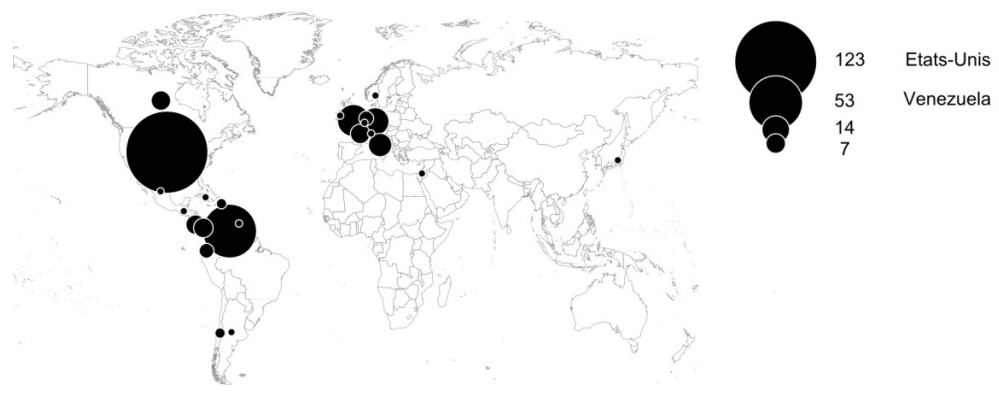

Champ : 1029 Colombiens.

Réalisation : C. Sierra-Paycha, Philcarto, 2015.

Ces résultats nous incitent à dépasser le paradigme du "couple migratoire "(Simon, 2002) pour comprendre les dynamiques migratoires contemporaines dont la migration colombienne est un exemple. Étudier de façon segmentée les migrants colombiens dans leurs différents pays d'émigration semble constituer une aporie. En effet, la porosité entre les champs migratoires 
semble être dynamisée par les dynamiques familiales, ici " photographiées " de manière transversale par la géographie résidentielle familiale des migrants.

\section{Un effet du genre sur l'espace résidentiel de la famille en contexte migratoire}

En général, les études sur la proximité de la parentèle montrent qu'il y a peu d'effet du sexe sur cette proximité que ce soit dans des contextes français (Maison et Ortalda, 1998) ou colombien (Dureau, 2004).

\section{Proximité du conjoint et des enfants pour les femmes migrantes}

On observe ici au contraire un effet important du sexe sur la localisation résidentielle de la famille (Tableau 2). Les femmes en couple cohabitent plus souvent avec leur conjoint (90\%) et corésident plus souvent avec leurs enfants (50\%) que les hommes (qui ne sont que $78 \%$ à vivre avec leur conjoint et $43 \%$ à vivre avec leurs enfants). Au-delà du logement, les enfants et les conjoints des migrantes sont plus souvent en Espagne (93\% des conjoints et $63 \%$ des enfants) que les conjoints et enfants des migrants hommes (respectivement $83 \%$ et $59 \%$ ). On peut formuler ici I'hypothèse d'un engagement différencié des hommes et des femmes dans la vie familiale. Les recherches sur le transnationalisme familial n'ont d'ailleurs pas manqué de souligner l'attachement de mères migrantes à avoir les enfants à proximité, et lorsque cela n'est pas possible, à mettre en place des stratégies de circulation entre les deux pays de résidence (Yépez et al., 2011).

Tableau 2 : Répartition des lieux de résidence des membres de la famille des migrants colombiens en Espagne selon le sexe et la nature du lien familial (en \%)

\begin{tabular}{|c|c|c|c|c|c|c|}
\hline Femmes $N=691$ & Conjoint & Enfants & Père & Mère & Fratrie & Total \\
\hline Espagne & 92,5 & 63,1 & 42 & 40 & 18,7 & 36,4 \\
\hline Colombie & 7,5 & 33,5 & 56,5 & 60 & 75,4 & 59,8 \\
\hline Pays tiers & 0 & 3,4 & 1,5 & 0 & 5,9 & 3,8 \\
\hline Total colonne & 100 & 100 & 100 & 100 & 100 & 100 \\
\hline Effectifs liens familiaux & 305 & 895 & 584 & 675 & 2643 & 5102 \\
\hline Hommes $N=338$ & Conjoint & Enfants & Père & Mère & Fratrie & Total \\
\hline Espagne & 83,3 & 58,8 & 43,1 & 42,9 & 25,8 & 39,4 \\
\hline Colombie & 16,7 & 38,3 & 54,2 & 57,1 & 68,4 & 56,8 \\
\hline Pays tiers & 0 & 2,9 & 2,7 & 0 & 5,8 & 3,8 \\
\hline Total colonne & 100 & 100 & 100 & 100 & 100 & 100 \\
\hline Effectifs liens familiaux & 150 & 379 & 295 & 308 & 1176 & 2308 \\
\hline
\end{tabular}

Lecture : Parmi les pères vivants des migrantes colombiennes en 2007, $37 \%$ vivent chez ces migrantes, $42 \%$ vivent en Espagne, 56,5 \% en Colombie et 1,5\% dans un pays tiers.

\section{Proximité de la fratrie pour les hommes migrants}

Les parents vivent légèrement plus près du logement des migrants hommes que de celui des migrantes ( $43 \%$ des pères et mères vivent en Espagne pour les 
hommes contre $42 \%$ et $40 \%$ pour les femmes), mais les différences semblent faibles. La fratrie a tendance à être plus proche du logement des hommes migrants que de celui des femmes migrantes. Pour les hommes, $6 \%$ de la fratrie coréside, $17 \%$ vit dans le même municipe et $26 \%$ vit en Espagne alors que pour les femmes, seulement $3 \%$ de la fratrie coréside, $9 \%$ vit dans le même municipe et $19 \%$ vit en Espagne.

\section{La fratrie : ancrage en Colombie et ouverture à d'autres espaces nationaux}

Le corollaire de ces tendances différenciées à la proximité résidentielle de la famille selon le sexe du migrant et la nature du lien est une présence plus importante des conjoints et enfants en Colombie chez les hommes (17\% des conjoints et $38 \%$ des enfants des hommes vivent en Colombie versus $7 \%$ des conjoints et $33 \%$ des enfants des femmes); une présence plus importante des parents et surtout de la fratrie en Colombie chez les femmes (57 \% des pères, $60 \%$ des mères et $75 \%$ de la fratrie des femmes vivent en Colombie versus $54 \%$ des pères, $57 \%$ des mères et $68 \%$ de la fratrie chez les hommes).

L'ancrage familial en Colombie est donc davantage du fait de la fratrie que de n'importe quel autre lien familial. D'après le tableau 1, 64 \% des liens familiaux résidant en Colombie sont composés par la fratrie des migrants alors que ces liens de fratrie ne représentent que $51 \%$ des liens familiaux. Par ailleurs, plus de la moitié des parents vivent en Colombie. C'est également la fratrie qui assure la connexion avec d'autres espaces nationaux en dehors du couple migratoire, $79 \%$ des liens familiaux résidant dans un pays tiers sont des liens de fratrie (alors que ceux-ci ne représentent que $51 \%$ des liens familiaux). C'est aussi parmi les liens de fratrie que la part des résidents dans un pays tiers est la plus forte ( $6 \%$ des frères, $3 \%$ des enfants, $2 \%$ des pères, vivent dans un pays tiers). La présence d'un membre de la famille à l'étranger est de surcroit très sélective puisqu'aucun migrant enquêté n'a un conjoint ou une mère résidant dans un pays tiers.

On constate de fortes disparités en termes de proximité résidentielle selon le sexe du migrant, alors que les conjoints et les enfants vivent plus près du domicile des femmes migrantes que de celui des hommes migrants, ce sont au contraire les parents et la fratrie qui vivent plus près du logement des hommes migrants. L'ancrage en Colombie est assuré, pour les deux sexes, essentiellement par la fratrie et par les parents et l'ouverture de l'espace résidentiel familial à d'autres espaces nationaux est très sélective et assurée surtout par la fratrie et dans une moindre mesure par les enfants et le père.

\section{Des espaces résidentiels familiaux différenciés selon les classes sociales}

Traditionnellement, les classes supérieures ont un espace de dispersion familiale plus élargi (Maison et Ortalda, 1998) maîtrisant ainsi, en tant qu'entité familiale, un plus grand nombre de lieux, en particulier dans le cas colombien (Dureau, 2004). Qu'en est-il en contexte migratoire ? La taille des parentèles n'est pas la même selon la classe sociale, appréhendée ici par le niveau d'études (la profession pouvant être trompeuse en migration). Dans la comparaison des plus qualifiés (ceux qui ont effectué des études supérieures) et des moins 
qualifiés (ceux qui ont effectué au maximum des études primaires) ${ }^{8}$, la taille de la famille apparaît plus réduite chez les plus qualifiés $(6,7$ membres en moyenne) que chez les moins qualifiés ( 8 membres). Dans la comparaison des migrants selon le niveau d'études, on considère qu'il n'y a pas d'effet de l'âge ni du sexe, car les âges médians et moyens, minimums maximums ainsi que les sex-ratios sont analogues.

\section{Des migrants très qualifiés proches de leur conjoint et de leurs enfants}

Tableau 3 : Répartition des lieux de résidence des membres de la famille des migrants colombiens en Espagne selon le niveau d'études et la nature du lien familial (en \%)

\begin{tabular}{|c|c|c|c|c|c|c|}
\hline $\begin{array}{l}\text { Éducation primaire } \\
N=174 \text { enquêtés }\end{array}$ & Conjoint & Enfants & Père & Mère & Fratrie & Total \\
\hline Espagne & 79,7 & 55 & 48,3 & 43 & 17,3 & 34,3 \\
\hline Colombie & 20,3 & 43,5 & 50 & 57 & 78,1 & 62,7 \\
\hline Pays tiers & 0 & 1,5 & 1,7 & 0 & 4,6 & 3 \\
\hline Total colonne & 100 & 100 & 100 & 100 & 100 & 100 \\
\hline Effectifs liens familiaux & 69 & 329 & 120 & 142 & 753 & 1413 \\
\hline $\begin{array}{l}\text { Éducation supérieure } \\
N=185 \text { enquêtés }\end{array}$ & Conjoint & Enfants & Père & Mère & Fratrie & Total \\
\hline Espagne & 96,9 & 75,5 & 39,1 & 36,6 & 15,9 & 35,5 \\
\hline Colombie & 3,1 & 16,8 & 56,4 & 63,4 & 76,1 & 58,7 \\
\hline Pays tiers & 0 & 7,7 & 4,5 & 0 & 8 & 5,8 \\
\hline Total colonne & 100 & 100 & 100 & 100 & 100 & 100 \\
\hline Effectifs liens familiaux & 98 & 155 & 156 & 175 & 661 & 1245 \\
\hline
\end{tabular}

Lecture : Parmi les conjoints des migrants colombiens n'ayant effectué aucune étude ou au maximum des études primaires en $2007,77 \%$ vivent chez ces migrants, $80 \%$ vivent en Espagne et $20 \%$ vivent en Colombie. En revanche, $92 \%$ des conjoints des migrants ayant fait des études supérieures vivent chez ces migrants, $97 \%$ de ces conjoints vivent en Espagne et seulement $3 \%$ vivent en Colombie.

Plus les migrants sont diplômés (Tableau 3), plus les couples cohabitant sont nombreux $(77 \%$ des conjoints corésidents chez les personnes ayant fait des études primaires contre $92 \%$ chez les personnes ayant fait des études supérieures). Seulement 80 \% des conjoints des moins qualifiés vivent en Espagne contre $97 \%$ des conjoints des migrants très qualifiés. On retrouve la même logique de proximité résidentielle selon le niveau d'études pour les enfants. Peu corésidents chez les moins qualifiés (36\%), ils le sont majoritairement chez les plus qualifiés (54\%). Seulement $55 \%$ des enfants des moins qualifiés vivent en Espagne contre $76 \%$ des enfants des plus qualifiés. En revanche, les

8 Les niveaux d'études intermédiaires occupent globalement des positions intermédiaires entre ces deux extrêmes du point de vue de la localisation résidentielle de la famille. 
parents vivent dans des résidences beaucoup plus proches des migrants moins qualifiés que des migrants plus qualifiés (46 \% des pères, $35 \%$ des mères des moins qualifiés corésident avec eux alors que chez les plus qualifiés, ces taux descendent respectivement à $31 \%$ et $26 \%$ ).

\section{Un ancrage familial en Colombie plus important chez les migrants peu qualifiés}

L'ancrage familial dans le pays d'origine est plus important pour les moins qualifiés, en effet $63 \%$ de la famille réside en Colombie contre $59 \%$ chez les plus qualifiés. La différence est faible, mais l'ancrage en Colombie n'est pas dévolu aux mêmes liens familiaux selon la classe sociale. L'ancrage en Colombie est plutôt assuré par les conjoints et les enfants chez les moins qualifiés $(20 \%$ de leurs conjoints y vivent contre $3 \%$ chez les plus qualifiés ; $43 \%$ des enfants y vivent contre $17 \%$ chez les plus qualifiés). Chez les plus qualifiés, I'ancrage en Colombie est davantage dévolu aux parents. $56 \%$ de leurs pères et $63 \%$ de leurs mères y vivent alors que ces proportions s'élèvent à $50 \%$ et $57 \%$ chez les moins qualifiés. De même, 27 \% des liens familiaux vivant en Colombie sont des liens parentaux chez les plus qualifiés contre $16 \%$ des liens pour les moins qualifiés ${ }^{9}$.

\section{Des espaces familiaux plus internationalisés pour les migrants très qualifiés}

L'ouverture à d'autres espaces nationaux est plus fréquente chez les plus qualifiés (6\%) que chez les moins qualifiés (3\%). Alors que chez les moins qualifiés, c'est la fratrie qui assure $83 \%$ de cette ouverture, chez les plus qualifiés, cette contribution de la fratrie diminue (74 \%) au profit du rôle des enfants ( $17 \%$ versus $12 \%$ chez les moins qualifiés) et du père ( $10 \%$ versus $4 \%$ chez les moins qualifiés $)^{10}$. Les enfants des migrants ayant réalisé des études supérieures résident proportionnellement plus dans un pays tiers ( $8 \%$ versus $1 \%$ ) tout comme les pères ( $4 \%$ versus $2 \%$ ) et la fratrie ( $8 \%$ versus $5 \%$ ) que ceux des migrants ayant réalisé uniquement des études primaires.

Les migrants très qualifiés se caractérisent donc par une proximité résidentielle plus forte du conjoint et des enfants alors que les migrants très peu qualifiés connaissent, au contraire, une proximité résidentielle plus forte des parents. II s'agit donc de migrations familiales dans le cas des plus qualifiés. L'ancrage familial en Colombie est plus important chez les moins qualifiés alors que l'ouverture à d'autres espaces nationaux est plus importante chez les plus qualifiés ; mais ces fonctions d'ancrage et d'ouverture n'incombent pas aux mêmes liens familiaux selon la classe sociale. L'ancrage en Colombie est davantage assuré par les enfants et les conjoints pour les moins qualifiés alors qu'il est davantage assuré par les parents pour les plus qualifiés. L'ouverture de l'espace résidentiel familial à d'autres espaces migratoires est en revanche davantage due à la fratrie pour les moins qualifiés alors que cette ouverture est plutôt due aux enfants et au père du migrant chez les plus qualifiés.

9 Ces chiffres ne figurent dans aucun tableau reproduit dans l'article. Il s'agit des pourcentages en ligne du tableau 3.

10 lbid. 
Une caractéristique des espaces résidentiels familiaux émergeant ici est celle d'une organisation transnationale différenciée selon la classe sociale. Les Colombiens les moins qualifiés apparaissent comme plus ancrés en Colombie (67\% des espaces résidentiels familiaux suivent une logique d'organisation binationale contre seulement $54 \%$ des espaces résidentiels familiaux des migrants très qualifiés) alors que les Colombiens les plus qualifiés connaissent des espaces résidentiels familiaux plus souvent trinationaux ( $26 \%$ des familles des migrants très qualifiés sont dispersés sur trois pays contre seulement $14 \%$ des familles des migrants les moins qualifiés) ${ }^{11}$. On retiendra donc une opposition entre des migrations familiales des classes supérieures au sein de configurations familiales multipolaires et des migrations plutôt isolées pour les classes populaires générant des organisations familiales bipolaires.

\section{L'espace résidentiel familial : un indicateur du degré d'installation}

La localisation résidentielle de la famille suit des logiques différentes selon le sexe du migrant et sa classe sociale ; elle reflète également l'année d'arrivée du migrant colombien en Espagne (Tableau 4). La part de la famille corésidente augmente faiblement avec la durée de l'étape migratoire en Espagne passant de $23 \%$ pour les migrants les plus récents à $27 \%$ pour les migrants les plus anciens ; la proximité de la famille augmente plus significativement avec la durée de l'étape migratoire lorsqu'on s'intéresse à la part des liens familiaux qui réside en Espagne, de $32 \%$ pour les migrants les plus récents, elle passe à $50 \%$ pour les migrants les plus anciens. Ces tendances ne s'observent significativement qu'avec la proximité résidentielle du conjoint et des enfants. Alors que seulement $61 \%$ des conjoints des migrants les plus récents vivent avec eux et que seulement $68 \%$ d'entre eux vivent en Espagne, $94 \%$ des conjoints des migrants les plus anciens résident avec eux et $99 \%$ vivent en Espagne. De même, la part des enfants corésidents ( $24 \%$ ) et des enfants vivant en Espagne $(29 \%)$ est très faible pour les migrants les plus récents alors que $56 \%$ des enfants des migrants les plus anciens vivent avec eux et $85 \%$ d'entre eux vivent en Espagne.

La conséquence de ces tendances est une diminution progressive de la part de la famille maintenant l'ancrage en Colombie avec la durée de la migration, $62 \%$ de la famille vit en Colombie pour les migrants les plus récents alors que cette part diminue à hauteur de $43 \%$ pour les migrants les plus anciens.

L'influence de la durée de la migration sur l'espace résidentiel de la famille montre comment une entrée par l'espace familial résidentiel complexifie la temporalité de la migration. D'une part, la localisation résidentielle de la famille opère comme indicateur du degré d'installation du migrant, mais d'autre part la localisation de l'ensemble de l'espace de vie est relativement inerte par rapport au transfert du simple lieu de résidence, à titre d'exemple, on constate que même parmi les migrants les plus anciens, une partie non négligeable du réseau familial (supérieure à $40 \%$ ) est toujours ancrée en Colombie.

11 Ces chiffres ne figurent dans aucun tableau reproduit dans l'article. II s'agit de la répartition des migrants selon le niveau d'études et la forme du réseau familial. 
Tableau 4 : Répartition des lieux de résidence des membres de la famille des migrants colombiens en Espagne selon la nature du lien familial et l'année d'arrivée (en \%)

\begin{tabular}{|c|c|c|c|c|c|c|c|}
\hline \multicolumn{2}{|l|}{$N=1029$} & Conjoint & Enfants & Père & Mère & Fratrie & Total \\
\hline \multirow{2}{*}{$\begin{array}{l}\text { Moins de } \\
2 \text { ans }\end{array}$} & Espagne & 67,7 & 28,7 & 39,9 & 35,5 & 24 & 31,7 \\
\hline & Colombie & 32,3 & 63,3 & 58,7 & 64,5 & 65,6 & 61,9 \\
\hline \multirow[t]{3}{*}{$N=92$} & Pays tiers & 0 & 8 & 1,4 & 0 & 10,4 & 6,4 \\
\hline & Total colonne & 100 & 100 & 100 & 100 & 100 & 100 \\
\hline & Liens vivants & 31 & 87 & 70 & 76 & 221 & 485 \\
\hline \multirow[t]{2}{*}{3 à 4 ans } & Espagne & 80 & 50,4 & 43,8 & 38,3 & 19 & 33,6 \\
\hline & Colombie & 20 & 44,6 & 56,2 & 61,7 & 75 & 62,3 \\
\hline \multirow[t]{3}{*}{$N=80$} & Pays tiers & - & 5 & - & - & 6 & 4,1 \\
\hline & Total colonne & 100 & 100 & 100 & 100 & 100 & 100 \\
\hline & Liens vivants & 35 & 121 & 64 & 68 & 316 & 604 \\
\hline \multirow[t]{2}{*}{5 à 6 ans } & Espagne & 89,4 & 55 & 37,9 & 32,8 & 16,4 & 32 \\
\hline & Colombie & 10,6 & 44,1 & 60,6 & 67,2 & 79,3 & 65,4 \\
\hline \multirow[t]{3}{*}{$N=366$} & Pays tiers & 0 & 0,9 & 1,5 & 0 & 4,3 & 2,6 \\
\hline & Total colonne & 100 & 100 & 100 & 100 & 100 & 100 \\
\hline & Liens vivants & 160 & 433 & 325 & 347 & 1359 & 2624 \\
\hline \multirow[t]{2}{*}{7 à 8 ans } & Espagne & 91,6 & 66,1 & 42,5 & 44,8 & 23,2 & 40,3 \\
\hline & Colombie & 8,4 & 31,5 & 56 & 55,2 & 72,4 & 57 \\
\hline \multirow[t]{3}{*}{$N=305$} & Pays tiers & - & 2,4 & 1,5 & - & 4,4 & 2,7 \\
\hline & Total colonne & 100 & 100 & 100 & 100 & 100 & 100 \\
\hline & Liens vivants & 130 & 384 & 275 & 323 & 1103 & 2215 \\
\hline \multirow[t]{2}{*}{$\begin{array}{l}9 \text { ans et } \\
\text { plus }\end{array}$} & Espagne & 99 & 84,6 & 52,5 & 53,6 & 29,6 & 50 \\
\hline & Colombie & 1,1 & 10 & 42,5 & 46,4 & 60 & 43,1 \\
\hline \multirow[t]{3}{*}{$N=179$} & Pays tiers & 0 & 5,4 & 5 & 0 & 10,4 & 6,9 \\
\hline & Total colonne & 100 & 100 & 100 & 100 & 100 & 100 \\
\hline & Liens vivants & 94 & 241 & 139 & 164 & 684 & 1322 \\
\hline
\end{tabular}

Lecture : Parmi les quatre-vingt-douze Colombiens arrivés le plus récemment en Espagne, c'est-à-dire moins de deux ans avant l'enquête, seulement trente-et-un ont un conjoint au moment de l'enquête. Si $61,3 \%$ de ces conjoints vivent dans le même logement, $32,3 \%$ vivent encore en Colombie. 
La distance intra-familiale est donc plus ou moins grande selon les caractéristiques sociales des migrants. Elle est plus grande chez les migrants récemment arrivés, chez les migrants les moins qualifiés et chez les hommes isolés de leur réseau familial. Au contraire, cette distance est moindre chez les migrants plus qualifiés, les migrants anciens et les femmes qui disposent de davantage de moyens de migrer en famille, ou d'avoir la famille à proximité. Lorsque la famille vit à très longue distance des migrants, il s'agit dans presque tous les cas d'un lieu de résidence en Colombie, car $94 \%$ des lieux de résidence de la famille ne résidant pas en Espagne se trouvent en Colombie (Tableau 1). Les descriptions des espaces résidentiels familiaux exposés en première partie valident I'hypothèse d'une forte dispersion des familles et invitent à s'intéresser dans la partie suivante aux flux qui relient ces différents lieux de résidence.

\section{Les interactions familiales à distance : communication, remise et circulation}

L'objectif de cette troisième partie est d'interroger la pertinence de l'hypothèse de la non-segmentation malgré la distance familiale, de se demander si le nouveau lieu de résidence du migrant est en relation avec les autres lieux de résidence de l'espace résidentiel familial, en particulier, les lieux de résidence en Colombie. Les études sur les systèmes résidentiels familiaux des années 1980 ont montré que les interactions privilégiées pour parler d'un système résidentiel étaient en premier lieu la mobilité, la communication et les solidarités économiques entre ces lieux (Osmont, 1987). Le champ des études migratoires a intégré ces éléments sous le terme générique de circulation migratoire, à savoir l'ensemble des mobilités générées par la migration, circulation de biens, de services, de personnes, mais aussi de symboles (Doraï et al., 1998). Nous nous efforcerons donc d'éprouver la validité de chacune de ces dimensions afin de comprendre si ces espaces résidentiels familiaux sont bien des systèmes résidentiels familiaux.

\section{Le maintien du contact à distance avec la famille en Colombie}

En contexte migratoire, la distance ne permet évidemment pas aux individus de se voir physiquement au quotidien. Pour maintenir les liens familiaux, les migrants mettent en place des stratégies de communication via les moyens usuels de communication disponibles à la fin des années 2000.

Dans notre étude (Tableau 5), l'immense majorité des migrants colombiens, $97 \%$, déclarent maintenir un contact répété avec de la famille ou des amis restés en Colombie. Le moyen privilégié pour contacter ses proches en Colombie est le téléphone dans $99 \%$ des cas et Internet (courriel et chat) dans $45 \%$ des cas. La communication repose d'ailleurs quasi exclusivement sur ces deux moyens puisque les autres moyens de communication (courrier postal, contact par personne interposée, etc.) sont utilisés par moins de $10 \%$ des migrants. D'après les études faites sur I'ENI, parmi l'ensemble des migrants en Espagne, ce sont les groupes sud-américains qui maintiennent le plus fréquemment le contact avec le pays d'origine (Gaete Quezada et Rodríguez Sumaza, 2010). Cependant, il ne s'agit pas d'interactions quotidiennes. En effet, quotidiennement, seulement $16 \%$ des migrants téléphonent à leur famille en Colombie et un quart lui envoie 
des mails. La temporalité de ces communications est davantage hebdomadaire (76\% des communications téléphoniques, $71 \%$ des échanges de courriels) ou mensuelle (99\% des échanges téléphoniques et $96 \%$ des courriels sont envoyés au moins une fois par mois).

Tableau 5 : Modes et fréquences de communications des migrants colombiens en Espagne avec la famille ou les proches en Colombie

\begin{tabular}{|c|c|c|c|c|c|c|c|}
\hline $\begin{array}{l}\text { Mode de } \\
\text { communication }\end{array}$ & \multirow{2}{*}{$\%$} & \multicolumn{6}{|c|}{$\%$ cumulé } \\
\hline $\begin{array}{l}\mathrm{N}=994 \text { Colombiens } \\
\text { en contact avec la } \\
\text { Colombie }\end{array}$ & & $\begin{array}{c}\text { Tous les } \\
\text { jours }\end{array}$ & $\begin{array}{l}\text { Toutes } \\
\text { les } \\
\text { semaines }\end{array}$ & \begin{tabular}{|c} 
Toutes \\
les deux \\
semaines
\end{tabular} & $\begin{array}{l}\text { Une } \\
\text { fois par } \\
\text { mois }\end{array}$ & $\begin{array}{l}\text { Une } \\
\text { fois } \\
\text { par an }\end{array}$ & $\begin{array}{l}\text { Moins } \\
\text { d'une } \\
\text { fois } \\
\text { par an }\end{array}$ \\
\hline Téléphone & 98,8 & 16,1 & 75,5 & 88,2 & 98,8 & 99,7 & 100 \\
\hline Courrier postal & 5,3 & \multicolumn{6}{|c|}{ ns } \\
\hline Courriel/chat & 45,2 & 25,4 & 70,8 & 82,2 & 95,8 & 99,6 & 100 \\
\hline $\begin{array}{l}\text { Par le biais d'autres } \\
\text { personnes }\end{array}$ & 2,9 & \multicolumn{6}{|c|}{ ns } \\
\hline Autre moyen & 0,7 & \multicolumn{6}{|c|}{ ns } \\
\hline
\end{tabular}

Lecture : Parmi les 994 Colombiens en contact régulier avec de la famille ou des proches en Colombie, 98,8 \% utilisent le téléphone et $45,2 \%$ utilisent le courriel ou le chat.16,1 \% des personnes contactant leurs proches par téléphone le font quotidiennement et 75,5 \% au moins une fois par semaine. Plusieurs réponses étant possibles, les individus pouvant communiquer par téléphone et par courrier, le total diffère de $100 \%$.

On remarque donc l'importance du recours aux moyens de communication modernes pour relier les familles à distance. Sans doute, dans des contextes passés, sans Internet et sans téléphone, la segmentation aurait été plus forte et les liens familiaux non entretenus. Même dans les cas où toute la famille du migrant vit en Espagne, le taux de communication avec la Colombie est élevé (85\%), mais moindre que dans les autres configurations (97\% des réseaux bipolaires, $98 \%$ des réseaux multipolaires et $100 \%$ des réseaux monopolaires colombiens communiquent régulièrement).

\section{La circulation entre l'Espagne et la Colombie}

Le maintien du lien familial ne saurait cependant se passer d'une rencontre en présence physique avec les autres membres de la famille. Toutes durées des étapes migratoires en Espagne confondues, un tiers des migrants n'est jamais retourné en Colombie depuis cette arrivée. Ce ratio de non-séjour en Colombie est relativement élevé et il faudrait l'éprouver à la lumière des durées des étapes migratoires en Espagne (Figure 7). Lorsque ces séjours ont lieu, ils se justifient en premier lieu pour rendre visite à de la famille ou des amis ( $86 \%$ des motifs de séjour). Ce motif peut se cumuler à d'autres motifs comme passer des vacances (64 \%) ou s'occuper des papiers en vue d'une régularisation en Espagne (8\%). Ces séjours, notamment en raison du prix du billet d'avion atteignant pratiquement le millier d'euros en août, sont donc importants par leur durée. $74 \%$ des 
séjours en Colombie durent un mois ou plus. L'entretien des réseaux familiaux représente donc un coût qui doit être amorti en restant suffisamment longtemps.

Quel que soit le type de réseau familial ayant encore des liens en Colombie (multipolaires, monopolaires colombiens et bipolaires), entre $66 \%$ et $70 \%$ des migrants sont déjà retournés, y compris parmi les réseaux multipolaires qui pourtant sont dispersés dans plusieurs pays, multipliant ainsi par le nombre de pays le coût d'entretien du réseau. En revanche, moins de la moitié des migrants dont toute la famille est en Espagne sont retournés en Colombie depuis leur arrivée en Espagne, ce qui montre l'importance de la présence de la famille en Colombie dans le maintien de la circulation avec la Colombie.

\section{L'envoi de remises financières}

Si la communication apparaît comme un phénomène assez généralisé, puisque $97 \%$ des Colombiens y recourent, le séjour dans le pays d'origine l'est moins (67\%). Qu'en est-il de l'envoi de remises financières, indicateur de stratégies de reproduction économique à l'œuvre malgré la distance ? Une étude sur les chaînes migratoires à partir d'ENI montre que seulement 49 \% des migrants envoient des remises (Ibid.). Le taux d'envoi de remises régulières de $65 \%$ montre que seulement une partie des migrants inscrivent a priori leur mobilité dans des stratégies de reproduction économique à proprement parler.

Lorsque cet envoi a lieu, il s'agit davantage d'une affaire de mois (68\% envoient leur remise au moins une fois par mois) que de semaines (seulement $1 \%$ envoient des remises toutes les semaines). Si la plupart envoient au moins une fois par an ( $86 \%)$, I'envoi de remises se fait également parfois de façon aléatoire selon les opportunités économiques de leur revenu, $14 \%$ des Colombiens enquêtés déclarent envoyer de l'argent quand ils le peuvent.

Tableau 6 : Montants des remises envoyées selon le niveau d'études (en €)

\begin{tabular}{|l|c|c|c|c|}
\hline $\begin{array}{l}\text { Niveau d'éducation } \\
\text { et remise d'argent } \\
\mathrm{N}=1029\end{array}$ & $\begin{array}{c}\text { \% des } \\
\text { migrants qui } \\
\text { envoient de } \\
\text { I'argent }\end{array}$ & $\begin{array}{c}\text { Montant } \\
\text { moyen } \\
\text { envoyé par } \\
\text { an }\end{array}$ & $\begin{array}{c}\text { Montant } \\
\text { médian } \\
\text { envoyé par } \\
\text { an }\end{array}$ & $\begin{array}{c}\text { Montant } \\
\text { maximum } \\
\text { envoyé par } \\
\text { an }\end{array}$ \\
\cline { 1 - 1 } Primaire & 64,9 & 2027 & 1200 & 12000 \\
\cline { 1 - 1 } $1^{\text {1er }}$ cycle secondaire & 60,7 & 2913 & 2000 & 12000 \\
\cline { 1 - 1 } $2^{\text {nd }}$ cycle secondaire & 71,5 & 1980 & 1500 & 15000 \\
\cline { 1 - 1 } Supérieur & 51,4 & 2669 & 2000 & 15000 \\
\cline { 1 - 1 } Total & 65,1 & 2239 & - & 15000 \\
\hline
\end{tabular}

Lecture : Seulement $51,4 \%$ des migrants ayant effectué des études supérieures envoient de l'argent régulièrement en Colombie. En moyenne, ils envoient 2669 euros et au maximum 15000 euros par an.

La plupart des migrants colombiens ont une idée du montant qu'ils envoient (79\%). En moyenne les remises s'élèvent à 2239 euros annuels avec des variations selon le niveau d'études (Tableau 6). La proportion de migrants envoyant des remises suit globalement une courbe en U. Si les migrants très peu qualifiés 
sont plus nombreux à envoyer des remises (65\%) que les migrants très qualifiés (51\%), ce sont les migrants ayant effectué une éducation de second cycle secondaire qui sont les plus nombreux à envoyer de l'argent (71\%). En dépit d'une plus faible propension des très qualifiés à envoyer de l'argent en Colombie, les montants moyen, médian et maximum des remises annuelles sont cependant plus élevés chez les plus qualifiés (2 669, 2000 et 15000 euros pour les plus qualifiés versus 2 027, 1200 et 12000 euros pour les moins qualifiés). Cependant, cette affirmation est à nuancer, car le groupe des migrants ayant fait le premier cycle d'études secondaires est celui qui envoie le plus d'argent annuellement en moyenne.

Lorsque l'on s'intéresse aux destinataires des remises envoyées par les migrants colombiens en Espagne (Tableau 7), ce sont les parents qui arrivent en tête des destinataires (63\% des migrants envoient de l'argent à leurs parents) suivis de la fratrie $(26 \%)$ et des enfants ( $26 \%)$. En raison d'une proximité résidentielle forte des conjoints, le taux de migrants envoyant de l'argent à leur conjoint est très faible $(6 \%)$. Les migrants envoyant des remises à des destinataires en dehors de la cellule familiale telle que nous l'avons défini dans cet article (conjoint, enfants, parents et fratrie) ne sont que $13,1 \%$ ce qui montre la validité du périmètre familial que nous avons défini plus haut dans les stratégies de reproduction économique de la famille. II serait intéressant ici d'observer au sein des différents liens familiaux transnationaux la fréquence de l'envoi de remise selon leur localisation. Si nous rapportons les envois de remise aux différents liens aux populations concernées par la présence de ces liens en Colombie, la hiérarchie des liens des solidarités économiques familiales est différente puisqu'y priment les liens intergénérationnels sur les liens collatéraux. $79 \%$ des migrants ayant des enfants résidant en Colombie envoient de l'argent régulièrement à leurs enfants en Colombie. $76 \%$ de ceux qui ont des parents en Colombie leur envoient de l'argent, alors que seulement $60 \%$ des migrants ayant un conjoint en Colombie leur transfèrent régulièrement des sommes et $31 \%$ des migrants ayant au moins un frère en Colombie leur envoient de l'argent.

Tableau 7 : Envoi de remises selon les types de destinataire (en \%)

\begin{tabular}{|l|c|c|}
\hline Destinataire N =667 & Oui & $\begin{array}{c}\text { Oui parmi les personnes ayant } \\
\text { ce lien vivant en Colombie }\end{array}$ \\
\hline Conjoint & 5,7 & 59,1 \\
\hline Parents & 62,7 & 76,3 \\
\hline Enfants & 25,6 & 78,9 \\
\hline Frères et sœurs & 26,1 & 30,6 \\
\hline Autres parents & 10,9 & - \\
\hline Non parents ou institutions & 3,2 & - \\
\hline
\end{tabular}

Lecture : Parmi les 667 migrants envoyant régulièrement de l'argent en Colombie, le conjoint est le destinataire dans seulement $5,7 \%$ des cas. En revanche, pour les migrants dont le conjoint vit en Colombie, ce dernier est le destinataire dans $59,1 \%$ des cas. 
La proportion de personnes produisant des flux entre les lieux de résidence, quel que soit le contenu de ces flux (flux de personnes, d'argent, de communication) est toujours majoritaire, ces flux servent à entretenir le réseau familial, notamment la partie restée dans le pays d'origine. Cependant, cette circulation de flux représente un coût monétaire, mais aussi temporel, et on peut faire l'hypothèse d'une baisse de l'intensité de cette circulation avec la durée de l'étape migratoire en Espagne.

\section{Une baisse de la circulation avec la durée de l'étape migratoire?}

Plus haut, nous avons vu que la présence de la famille en Colombie avait tendance à diminuer avec la durée de l'étape migratoire en Espagne. Les migrants les plus anciennement installés ont en effet plus de famille en Espagne qu'en Colombie. Or, la famille est la principale raison des visites en Colombie, mais aussi le principal destinataire des transferts d'argent et le principal contact en Colombie lors des communications transnationales. Est-ce à dire que ces flux Espagne-Colombie diminuent également avec la durée de l'étape migratoire en Espagne, synonyme d'installation ? La figure 7 confirme cette baisse, pour les flux de communication : alors que $99 \%$ des migrants les plus récents communiquent régulièrement avec la Colombie, la part des migrants les plus anciens communiquant est plus faible (90\%). Les flux monétaires, quant à eux, connaissent une évolution légèrement différente avec une proportion de migrants remisant en Colombie qui augmente au début de l'étape migratoire (51\% pour les migrants arrivés un à deux ans avant l'enquête, puis $73 \%$ des migrants arrivés cinq à six ans avant l'enquête), mais qui diminue au-delà de six ans passés en Espagne (seulement $48 \%$ des migrants en Espagne depuis plus de neuf ans envoient de l'argent en Colombie). La proportion de migrants ayant effectué un séjour en Colombie ne fait qu'augmenter avec la durée de l'étape migratoire ; mais avec le temps, les séjours sont moins fréquents. En effet, le temps moyen (exprimé ici en mois) séparant le dernier séjour en Colombie du moment de l'enquête s'accroit avec la durée de l'étape migratoire : les migrants arrivés depuis neuf ans ou plus connaissent la durée moyenne maximale (cinquante-deux mois soit plus de quatre ans) séparant leur dernier séjour en Colombie de la date de l'enquête. La figure 7 montre une rupture commune aux tendances des trois types de flux au-delà de sept à huit ans passés en Espagne. II s'agit d'un effet lié à la composition de la cohorte la plus ancienne. Cette dernière cohorte rassemble des individus arrivés, certes pour la plupart dans les années 1990, mais pour certains avant les années 1980. Or ces derniers ont très peu de contact avec la Colombie. Leur faible implication dans les flux Espagne/Colombie entraîne donc une discontinuité dans les courbes, exagérant les tendances observées.

Ces résultats mettent en lumière une modification de l'intensité de la circulation avec la durée de l'étape migratoire qui rappelle la notion de situation de mobilité proposée par Imbert (2014). La " situation de mobilité " est amenée à évoluer au cours de la trajectoire des individus ; les pratiques de circulation peuvent ainsi caractériser un moment de la trajectoire de mobilité. De même, dans le cas des migrants colombiens en Espagne, la circulation, principalement intrafamiliale, semble être la plus intense lorsque les migrants sont suffisamment installés pour pouvoir mettre en place des pratiques de circulation (envois 
d'argent, et allers-retours Colombie-Espagne), mais également lorsqu'il reste encore des membres de la famille avec qui échanger en Colombie.

Figure 7 : Proportion de migrants colombiens en Espagne en situation de " circulation " avec la Colombie selon la durée de l'étape migratoire en Espagne

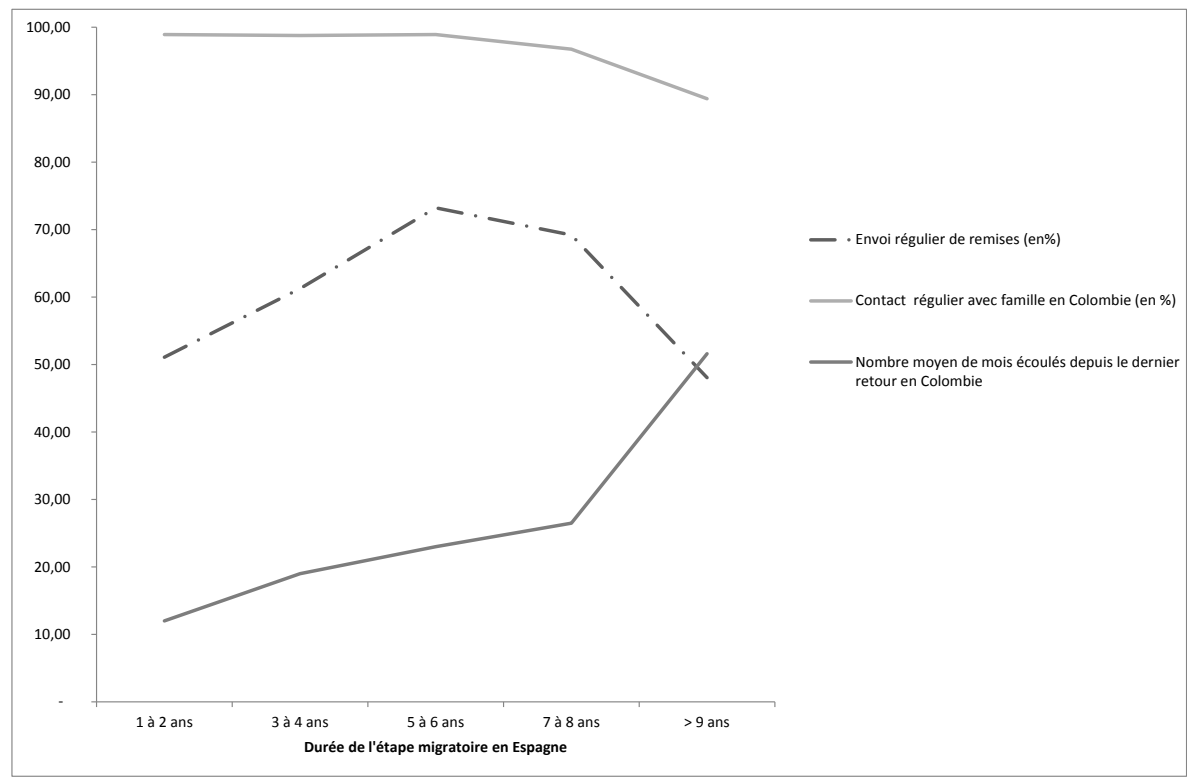

\section{Conclusion}

L'objectif de cet article était double. II s'agissait d'abord de resituer le lieu de résidence du migrant colombien dans l'espace résidentiel de sa famille en faisant varier l'âge, le sexe, le niveau d'études et l'année d'arrivée. Ensuite, on s'est demandé si, malgré la distance, les lieux de résidence étaient encore en interaction ; ces échanges étant matérialisés par les communications, les mobilités des migrants et les remises selon le temps passé en migration.

Comme nous l'avons vu dans la seconde partie, la totalité de la famille n'est que rarement à proximité du migrant, seulement $8 \%$ des réseaux familiaux sont intégralement en Espagne. L'ancrage en Colombie est la principale cause de la distance intrafamiliale, car la présence de membres de la famille dans un pays hors du couple migratoire est somme toute minoritaire et l'apanage des familles des migrants les plus qualifiés. S'intéresser aux interactions familiales à distance des migrants colombiens en Espagne, c'est donc s'intéresser avant tout aux interactions familiales entre l'Espagne et la Colombie. C'est précisément ce qui a fait l'objet de notre attention dans la troisième partie. L'interaction familiale à distance, par la circulation, les remises d'argent ou la communication à distance est toujours majoritaire. À contre-courant de certains travaux évoquant un déclin relatif de la famille comme unité socio-économique de reproduction (Théry, 2001 ; Weber, 2013), les liens familiaux consanguins apparaissent ici au contraire comme des liens de solidarité économiques, en particulier dans le 
cas des relations intergénérationnelles (76\% des migrants envoient de l'argent à leurs parents en Colombie et $79 \%$ à leurs enfants), mais aussi collatérales (31 \% des migrants envoient de l'argent à leurs frères et sœurs en Colombie). Si les logiques d'interaction forte à distance semblent être majoritaires, elles ne sont pas pour autant universellement partagées. Dans des cas minoritaires, la segmentation familiale est un fait (plus aucun lien avec la Colombie).

Seulement $3 \%$ des migrants ne communiquent pas régulièrement avec la Colombie, $35 \%$ n'envoient pas d'argent régulièrement et $33 \%$ des migrants ne sont pas revenus en Colombie depuis leur arrivée en Espagne. Les périodes de circulation avec la Colombie sont toutefois à replacer dans la temporalité des parcours migratoires individuels; elles semblent en effet correspondre à des moments caractérisés de l'étape migratoire en Espagne, après quelques années de résidence en Espagne. La notion de système résidentiel familial apparaît ici comme une notion utile pour penser les migrations individuelles colombiennes. Cette notion permet donc de nuancer l'originalité de l'organisation des familles transnationales, la famille transnationale apparaît en effet comme un cas particulier du système résidentiel familial élargi où au moins deux lieux de résidence sont séparés par une frontière nationale, mais hormis une différence d'échelle, on ne note pas de différence de nature entre le système résidentiel familial tel qu'il a été découvert dans des contextes de migrations internes et la famille transnationale.

\section{Références bibliographiques}

Balán Jorge and Dandler Jorge (1987) Marriage process and household formation: Migration in the Cochabamba region (Bolivia) and Bolivian migrants in Buenos Aires (Argentina), Lomé, CRDI-ORSTOM-URD.

Beltramone André (1975) Définition logique des flux migratoires intérieurs, in Migrations intérieures, méthodes d'observation et analyse, Paris, CNRS Éditions, pp. 491-518.

Bonvalet Catherine et Lelièvre Éva (2005) Les lieux de la famille, Espaces et sociétés, 120-121 (2), pp. 99-122.

Burawoy Michael (1976) The functions and reproduction of migrant labor: Comparative material from Southern Africa and the United States, American Journal of Sociology, 81 (5), pp. 1050-1087.

Colectivo IOÉ (2012) Impactos de la crisis sobre la población inmigrante, Madrid, Organización Internacional para las Migraciones, 189 p.

Courgeau Daniel (1975) Le concept de migration, Migrations, état civil, recensements administratifs, actes du IVème colloque de démographie africaine, Ouagadougou, Institut national de la statistique et de la démographie, pp. 27-32.

Domenach Hervé et Picouet Michel (1995) Les Migrations, Paris, Presses Universitaires de France, 128 p.

Doraï Mohamed Kamel, Hily Marie-Antoinette, Loyer Frantz et Ma Mung Emmanuel (1998) Bilan des travaux sur la circulation migratoire, Rapport final commandé par le ministère de la Solidarité et de l'Emploi, Direction de la Population et des Migrations (DPM), Poitiers, $145 \mathrm{p}$. 
Dureau Françoise (2004) Sistemas residenciales: conceptos y aplicaciones, Territorios, 11, pp. 41-70.

Dureau Françoise et Dupont Véronique (1994) Rôle des mobilités circulaires dans les dynamiques urbaines. Illustrations à partir de l'Équateur et de I'Inde, TiersMonde, 35 (140), pp. 801-829.

Dureau Françoise et Florez Carmen Elisa (1996) Dynamiques démographiques colombiennes : du national au local, in Jean-Michel Blanquier et Christian Gros Éds., La Colombie à l'aube du troisième millénaire, Paris, IHEAL, pp. 139-166.

Dureau Françoise et Giroud Matthieu (2014) La dynamique biographique des lieux de la circulation : une "affaire de famille "?, in Christophe Imbert et al. Éds., D'une métropole à l'autre. Pratiques urbaines et circulations dans l'espace européen, Paris, Armand Colin, pp. 305-363.

Echeverri de Ferrufino Ligia (2004) La familia de hecho en Colombia, Universitas Humanística, 14 (23), pp. 99-107.

Engels Friedrich (1976 [1884]) L'origine de la famille, de la propriété privée et de I'État, Préface de la première édition, Moscou, Éditions du Progrès, 318 p.

Gaete Quezada Ricardo y Rodríguez Sumaza Carmen (2010) Una aproximación al análisis de las cadenas migratorias en España a partir de la Encuesta Nacional de Inmigrantes, Revista de ciencia política (Santiago), 30 (3), pp. 697-721.

Golaz Valérie et Lelièvre Éva (2012) Mémoire, appréciation de la vie et entourage familial, in Catherine Bonvalet et Éva Lelièvre, De la famille à l'entourage. L'enquête. Biographies et entourage, Paris, Ined Éditions, pp. 95-110.

Gonzalez Fernando y Echeverri Beatriz (2009) Los inmigrantes colombianos en España, in David-Sven Reher y Miguel Requena Eds., Las múltiples caras de la inmigración en España, Madrid, Alianza Editorial, pp. 153-184.

Guarnizo Luis Eduardo (2006) The Economics of Transnational Living, International Migration Review, 37 (3), pp. 666-699.

Henry Louis (1981) Dictionnaire démographique multilingue, Liège, UIESP Odrina, $180 \mathrm{p}$.

Imbert Christophe, Dubucs Hadrien, Dureau Françoise et Giroud Matthieu (Éds.) (2014) D'une métropole à l'autre : pratiques urbaines et circulations dans l'espace européen, Paris, Armand Colin, $484 \mathrm{p}$.

Le Bris Émile, Osmont Annik, Marie Alain et Sinou Alain (1987) Famille et résidence dans les villes africaines. Dakar, Bamako, Saint-Louis, Lomé, Paris, L'Harmattan, $270 \mathrm{p}$.

Maison Dominique et Ortalda Laurent (1998) Morphologie spatiale de la parenté. Proximités géographiques au sein de la famille étendue selon l'enquête Proches et parents, in Yves Grafmeyer et Francine Dansereau Éds., Trajectoires familiales et espaces de vie en milieu urbain, Lyon, Presses Universitaires de Lyon, pp. 101-120.

Ma Mung Emmanuel (1999) La dispersion comme ressource, Cultures et Conflits, 33-34, pp. 89-103.

Osmont Annik (1987) Stratégies familiales, stratégies résidentiellęs en milieu urbain : un système résidentiel dans l'agglomération dakaroise, in Émile Le Bris et al., Famille et résidence dans les villes africaines. Dakar, Bamako, Saint-Louis, Lomé, Paris, L'Harmattan, pp. 115-176. 
Pfirsch Thomas (2009) Proximité familiale et organisation résidentielle de la parentèle dans les élites d'une ville d'Europe du Sud. L'exemple de Naples, Articulo - Journal of Urban Research, 1, [en ligne]. URL : https://articulo.revues. org/1052

Pumares Fernández Pablo, García Coll Arlinda y Asensio Hita Ángeles (2006) La movilidad laboral y geográfica de la población extranjera en España, Madrid, Observatorio Permanente de las Migraciones, Ministerio de Asuntos Sociales.

Quesnel André (2009) De la communauté territoriale à l'organisation familiale en archipel : la mobilité spatiale des familles rurales en Afrique de l'Ouest et au Mexique, in Françoise Dureau et Marie-Antoinette Hily Éds., Les mondes de la mobilité, Rennes, Presses Universitaires de Rennes, pp. 67-103.

Quesnel André et Del Rey Alberto (2004) Mobilité, absence de longue durée et relations intergénérationnelles en milieu rural (état du Veracruz, Mexique), Cahiers des Amériques Latines, 45, pp. 75-91.

Reher David-Sven y Requena Miguel (Eds.) (2009) Las múltiples caras de la inmigración en España, Madrid, Alianza Editorial, 321 p.

Robette Nicolas (2012) Les espaces de vie individuels : de la géographie à une application empirique en démographie, Cybergeo: European Journal of Geography, document 605.

Sierra-Paycha Celio (2014) La dinámica espacial y demográfica de un sistema migratorio en contexto de crisis: el caso de los colombianos en España a partir de la estadística de variaciones residenciales (2005-2012), Actes du XIV Congreso Nacional de Población, AGE, pp. 314-326.

Simon Gildas (2002) Les migrations internationales, Population et Sociétés, 382, pp. 1-4.

Théry Irène (2001) Le démariage : justice et vie privée, Paris, O. Jacob, 400 p.

Weber, Florence (2013) Penser la parenté aujourd'hui : la force du quotidien, Paris, Éditions Rue d'Ulm, Sciences sociales, 262 p.

Yépez Isabel, Ledo Carmen et Marzadro Mirko (2011) " Si tu veux que je reste ici, il faut que tu t'occupes de nos enfants! "Migration et maternité transnationale entre Cochabamba (Bolivie) et Bergame (Italie), Autrepart, 57-58 (1), pp. 199-213. 


\section{Celio Sierra-Paycha}

\section{La circulation au sein de l'espace résidentiel de la famille. Le cas des familles de migrants colombiens en Espagne}

La géographie de l'espace résidentiel des familles des migrants est un sujet rarement abordé de façon quantitative. À partir de l'analyse de données d'enquête concernant les migrants colombiens installés en Espagne (ENI, 2007), nous décrivons les formes tissées par les différents lieux de résidence des membres de la famille, que ceux-ci se trouvent en Espagne, en Colombie ou ailleurs dans le monde. La mise en relation des différents lieux de résidence familiaux y est interrogée sous l'angle des circulations; circulation des migrants eux-mêmes, flux monétaires et communication transnationale (par téléphone et par Internet), permettent de considérer ces géographies familiales comme de véritables "systèmes résidentiels familiaux ".

\section{Circulation into Family Residential Space. The Case of Colombian Migrant's Families in Spain}

The residential geography of families of migrants is a topic rarely addressed from a quantitative point of view. Based on the analysis of survey data on Colombian migrants in Spain (ENI, 2007), this article aims at describing the network build up by the different places of residence of those family members, may these be in Spain, Colombia or anywhere else in the world. The links between the different places of residence are handled means of the circulation approach. Migrant's circulation, but also monetary remittances and transnational communication by telephone or Internet, may help consider these family geographies as "family residential systems".

\section{Circulación en el espacio residencial de las familias. El caso de las familias de inmigrantes colombianos en España}

La geografía residencial de las familias de los inmigrantes es un tema poco estudiado con métodos cuantitativos. A partir del análisis de datos de encuesta sobre inmigrantes colombianos en España (ENI, 2007), este artículo propone describir las redes dibujadas por los distintos lugares de residencia de dichas familias, que estas se encuentren en España, en Colombia o en otra parte del mundo. Interrogamos la relación entre los distintos lugares de residencia mediante el enfoque de la circulación. La circulación de los inmigrantes, pero también de los flujos monetarios y de comunicación transnacional por teléfono o por Internet, permiten considerar estas geografías familiares como «sistemas residenciales familiares». 